\title{
EVOLUÇÃO DA MÃO DE OBRA OCUPADA NO SETOR AGROPECUÁRIO DO RIO GRANDE DO SUL ${ }^{1}$
}

\author{
Izete Pengo Bagolin ${ }^{2}$ \\ Valter José Stülp ${ }^{3}$
}

Resumo: O primeiro objetivo do presente trabalho é testar a existência de convergência dos níveis de ocupação da mão de obra entre as diferentes regiões do RS. Isso é feito utilizando matrizes de Markov. O segundo objetivo identifica, através de uma regressão em painel, as variáveis que auxiliam a explicar a evolução dos níveis de ocupação. Os resultados mostram que, no longo prazo, haveria pequeno aumento no percentual das regiões situadas nas duas classes intermediárias. Nas demais quatro classes, haveria, no futuro, uma pequena redução na participação percentual das regiões. As variáveis com impacto negativo sobre a ocupação da mão de obra são a participação de estabelecimentos com mais de duzentos hectares no total dos estabelecimentos; o nível de mecanização das lavouras; as lavouras de arroz, soja e feijão; e as participações da produção bovina e ovina na produção animal. A participação dos estabelecimentos rurais inferiores a vinte hectares, a igualdade na distribuição da área entre os estabelecimentos e as lavouras de fumo e de uvas apresentam impactos positivos.

Palavras Chave: Ocupação de mão de obra, regiões do RS, produção rural

Recebido em: 28/6/2012; Aceito em: 3/09/2012.

2 Professora do PPGE PUCRS. E-mail: izete.bagolin@pucrs.br

3 Professor do PPGE PUCRS. E-mail: vstulp@pucrs.br 


\begin{abstract}
Initially, using a Markov transition matrix, the study analyses the regional convergence of the state of Rio Grande do Sul to a similar level of rural labor occupied per hectare. The results indicate that there would occur a small increase in the percentage of regions in the class situated above the state average and in the one located below the state average. The percentage of the regions in the two classes situated at the most inferior levels and in the two classes at the most superior levels of labor occupation would reduce very little. In the second part, a regression analyses with panel data was used to examine the variables that would explain the evolution of the levels of labor occupation. The data are from 1975 and 2006 IBGE agricultural censuses. The participation of the rural establishments greater than two hundred hectares; the level of mechanization; the crops of rice, soybeans and beans; and the participation level of cattle and sheep in the livestock production presented negative impacts on labor occupation. The participation of the rural establishments smaller than twenty hectares; the greater equality in the area distribution among the rural establishments (lower GINI index); and the crops of tobacco and grapes presented positive effects.
\end{abstract}

Key Words: Occupation of labor, regions, rural production

\title{
1. Introdução
}

As questões relacionadas à mão de obra agrícola, à mobilidade de fatores e de pessoas, à produtividade do capital e trabalho e à migração têm sido objeto de estudo desde os modelos clássicos iniciados por Lewis (1954), Todaro (1969) e Harris \& Todaro (1970). Naquele primeiro estágio do debate, a migração rural-urbana parecia ser facilmente explicável pelos modelos clássicos de economia dual em que naturalmente a agricultura cumpria sua função de liberar mão de obra para a indústria.

No entanto, estes modelos sofreram críticas e perderam importância entre o final dos anos 1970 e início dos anos 1980. A partir de então, a busca pelos determinantes da mobilidade da mão de obra tem ampliado o campo de análise e, mais recentemente, a tese da interdependência entre migração, aglomeração e crescimento econômico tem conquistado adeptos. De acordo com o World Development Report (WORLD BANK, 2010), a migração interna e a aglomeração de mão de obra qualificada são fontes inesgotáveis de crescimento econômico e, além disso, contribuem 
para a convergência de renda, para aumentar o bem-estar e para garantir retornos do investimento em capital humano. Lucas Jr. (2004) afirma que as cidades são lugares onde novos migrantes podem acumular as habilidades necessárias e obter os ganhos da aglomeração. Segundo ele, mesmo nas economias pós-coloniais de crescimento rápido, a passagem de uma economia 90\% rural para uma 90\% urbana é apenas uma questão de décadas.

Mesmo assim, em muitas situações, a dinâmica da mão de obra, o envelhecimento da população rural, o esvaziamento do campo e o aumento do desemprego urbano ainda são considerados fenômenos preocupantes. Nestas situações, as variações na quantidade de mão de obra não são necessariamente um lubrificante para o crescimento econômico, não podendo ser consideradas "naturais" e nem sempre contribuindo para a convergência de renda e/ou de mão de obra entre as regiões.

Ainda de acordo com o World Development Report (WORLD BANK, 2010), a tese da migração como motor do crescimento, bem-estar e convergência só é verificável empiricamente quando os determinantes da mobilidade são racionais e baseados na busca por melhores oportunidades e retornos econômicos. Porém, quando a mobilidade é determinada pela falta de oportunidades e busca por lugares com maior oferta de serviços públicos, o resultado pode ser negativo, reduzindo ainda mais o bemestar e os ganhos econômicos agregados.

O ritmo, o estágio, os determinantes e a direção dos fluxos da força de trabalho variam de acordo com as particularidades, o estágio e as características do desenvolvimento de cada país ou região. Em outras palavras, os determinantes da mobilidade da mão de obra são heterogêneos e dinâmicos e por isso de difícil generalização.

As evidências empíricas mostram que países distintos em diferentes estágios de desenvolvimento e que, por consequência, são detentores de características particulares apresentam níveis e dinâmicas próprias de mão de obra. Por exemplo, Vaidyanathan (1986) destaca que na Índia 
o percentual da mão de obra - tanto masculina quanto feminina - que está efetivamente empregada na agricultura e atividades relacionadas apresentou redução ao longo de todo o período estudado pelo autor. De acordo com Irwin et al. (2010), na América do Norte, as tecnologias poupadoras de mão de obra e outras industrias de recursos naturais levaram à redução no uso de mão de obra rural, aumento na paridade da renda rural-urbana e diversificação da atividade rural. Stabler e Olfert (2009, apud Irwin et al., 2010) mostram que a parcela de mão de obra utilizada nas fazendas do Canadá se reduziu de 60\% em 1931 para 8\% em 2008.

Nos Estados Unidos, apesar da baixa utilização de mão de obra em atividades rurais, a quantidade de mão de obra no meio rural que desenvolve atividades não agrícolas tem se mantido estável. Ainda de acordo com Irwin et al. (2010), no início do século vinte, a parcela da população rural era de $42 \%$, a da população urbana, de $35 \%$, e a parcela da população rural não agrícola, de $23 \%$. Cem anos depois, a parcela da população rural não agrícola se manteve perto dos $20 \%$ e a população rural-agrícola se reduziu para aproximadamente $1 \%$.

A China apresentou dinâmica própria, tanto em resposta aos incentivos e restrições governamentais, quanto pelo efeito da revolução. As evidências mostram que a sensibilidade aos incentivos pró-migração rural urbana é maior que a sensibilidade às políticas restritivas. $\mathrm{O}$ retorno das pessoas ao meio rural só se verificou durante a contrarrevolução cultural, quando os intelectuais e a elite urbana retornaram ao campo. Excetuando-se esse período, o fluxo de mão de obra em direção às cidades tem se mantido ao longo do tempo, variando apenas em intensidade e condições econômicas do país (WORLD BANK, 2010).

O Rio Grande do Sul tem se mantido entre os estados brasileiros com participação importante na produção de alimentos para abastecimento das necessidades do país, com destaque para a produção de grãos especialmente soja, arroz e milho - e na produção pecuária - carne, leite e derivados - que são provenientes da grande empresa agrícola intensiva em tecnologia e poupadora de mão de obra. A utilização estadual de mão 
de obra rural se manteve estável com uma média de 0.99 equivalentes homem por hectare, de 1975 a 2006.

No entanto, a intensidade no uso de mão de obra difere tanto entre regiões quanto ao longo do tempo. Mesmo quando se mantém a análise agregada, é possível perceber que, apesar de a média ter se mantido constante, o desvio padrão e a variância apresentaram valores superiores em 2006 em comparação com 1975. Apenas para ilustrar que as variações não foram homogêneas entre as regiões, destaca-se que, entre as 156 regiões, 76 apresentaram redução no uso de mão de obra, 8 não apresentaram variação e 72 apresentaram aumento no uso de mão de obra.

A partir destas constatações, tem-se como objetivo analisar a dinâmica das variações no uso de mão de obra entre 1975 e 2006 e entre as regiões do estado do Rio Grande do Sul. O estudo está organizado em mais três seções, além desta introdução. Na próxima seção, são apresentados os procedimentos metodológicos para agrupamento das regiões, análise da convergência, identificação dos determinantes das variações no uso da mão de obra e fontes dos dados. Na seção seguinte, são apresentados e discutidos os resultados da análise de convergência e das regressões. Por fim, são apresentadas as considerações finais.

\section{Metodologia}

O primeiro objetivo do estudo é verificar como evolui a ocupação da mão de obra no setor agropecuário do RS, em termos de equivalentes homem por hectare explorado, ao longo do tempo. O segundo objetivo é analisar quais variáveis são responsáveis por esta evolução. Para atingir o primeiro objetivo, utiliza-se a metodologia de matrizes de Markov. A consecução do segundo objetivo é feita por uma regressão estatística. 


\subsection{Análise da convergência}

Para analisar a evolução da mão de obra ocupada no setor agropecuário do RS ao longo do tempo, foram utilizados os dados dos censos agropecuários de 1975 e 2006. Esse período foi escolhido por ser longo o suficiente para captar convergência de longo prazo, evitando, assim, que os resultados fossem contaminados por efeitos cíclicos de curto prazo. Em função disso, optou-se por não utilizar os dados do censo de 1995/1996. Para efetivar a análise proposta com base nos dados dos censos agropecuários de 1975 e 2006, é necessário que as regiões, nestes dois períodos que servem de base para o estudo, sejam geograficamente homogêneas. Em virtude do grande número de emancipações municipais ocorridas de 1975 ao início do presente milênio, houve necessidade de agregar municípios, constituindo regiões que fossem geograficamente homogêneas durante este período considerado. Há 156 regiões homogêneas de 1975 a 2006, sendo estas as consideradas no estudo.

Em relação a cada região, é calculada a mão de obra ocupada em termos de equivalentes homem por hectare explorado referente ao ano de 1975 e ao ano de 2006. Estes valores são expressos em termos relativos à média do Estado para cada um destes dois períodos. O Censo Agropecuário apresenta as categorias de pessoal ocupado pelos estabelecimentos agropecuários. Estas categorias são as seguintes: 1) responsável e membros não remunerados da família; 2) empregados permanentes; 3) empregados temporários; 4) parceiros; e 5) outra condição. Referentes a cada categoria, são informados o número de homens e de mulheres e o número de pessoas com 14 anos e mais de idade.

Para somar o pessoal ocupado das diversas categorias, procedeu-se à sua uniformização, transformando o número de pessoas em número de equivalentes homens. $\mathrm{O}$ equivalente homem corresponde a 300 dias de trabalho de um homem adulto. Para a transformação, utilizou-se a tabela referida por Guerreiro (1996). 
Como área de terra explorada, são consideradas as áreas de lavouras (permanentes e temporárias), de pastagens (naturais e plantadas) e de florestas plantadas. Não é considerada a área de matas ou florestas naturais.

Para a consecução do primeiro objetivo, isto é, analisar a evolução da ocupação da mão de obra ao longo do tempo, são consideradas as duas distribuições regionais de equivalentes homem por hectare explorado, a de 1975 e a de 2006, em valores relativos. Determina-se uma mesma estrutura de classes para estas duas distribuições. Com base nesta estrutura de classes, é possível examinar como as regiões migraram de uma classe para outra e assim construir uma matriz de probabilidades de transição das regiões entre classes. Esta é a matriz de probabilidades de transição de Markov.

Para a determinação da estrutura de classes, é necessário determinar uma dimensão do intervalo das classes, designada por $h$. Um $h$ muito grande faz com que haja um grande número de observações em cada intervalo de classe, diminuindo a variância da estimativa. No entanto, isto leva a um formato de histograma menos adequado, pois aumenta o viés da estimativa. Com um $h$ pequeno, ocorre o contrário: reduz-se o viés, mas aumenta-se a variância (Pagan e Ullah, 1999).

Portanto, o valor de $h$ deve ser escolhido de modo a resultar em um trade-off ótimo entre viés e variância da estimativa. Devroye e Györfi (1985, apud Magrini 1999, p.264) recomendam como o valor ótimo de $h$ o determinado pela fórmula $h=2,72 s n^{-1 / 3}$, em que $s$ é o desviopadrão da distribuição e $n$ o número de observações, neste caso, 156. Segundo os autores, esta expressão para encontrar o valor de $h$ seria adequada, principalmente, nos casos em que as observações seguissem uma distribuição normal.

Assim, antes da determinação da matriz de Markov, procede-se ao teste de normalidade de cada uma das duas distribuições de equivalentes homem por hectare explorado, em valores relativos, através do teste de Kolmogorov-Smirnov (SIEGEL, 1956). 
Dado o valor de $h$, constatada a normalidade de cada distribuição e determinadas as classes de equivalentes homem por hectare explorado, em termos relativos, verifica-se quantas regiões migraram de uma classe para outra, ou permaneceram na mesma classe entre o ano de 1975 e 2006. Assim, são determinados a matriz de probabilidades de transição (matriz de Markov) e o sistema de equações de diferenças cuja solução fornece as informações referentes ao processo de evolução das regiões quanto à ocupação da mão de obra no longo prazo.

\subsection{Regressão estatística.}

Na regressão estatística, destinada a analisar as variáveis explicativas dos níveis de ocupação da mão de obra, é utilizada a técnica de dados em painel. Esta técnica permite eliminar os efeitos fixos de certas características das unidades de observação que são as regiões, ao longo do tempo, e que não estão incluídas nas variáveis independentes consideradas. Assim, certas regiões podem apresentar características edafoclimáticas que influenciam o processo de produção e, portanto, a ocupação da mão de obra. Estes efeitos fixos podem ser representados por $a_{i}$ sendo que o subscrito i se refere à i-ésima região.

Referente ao ano de 1975, a função a estimar é como segue:

$$
\ln \mathrm{Y}_{\mathrm{i} 1}=\mathrm{b}_{0}+\mathrm{b}_{1} \mathrm{X}_{1 \mathrm{i} 1}+\mathrm{b}_{2} \mathrm{X}_{2 \mathrm{i} 1}+\mathrm{b}_{3} \mathrm{X}_{3 \mathrm{i} 1}+\ldots+\mathrm{b}_{\mathrm{k}} \mathrm{X}_{\mathrm{ki} 1}+a_{i}+\mu_{\mathrm{i} 1}
$$

$\mathrm{Y}_{\mathrm{i} 1}$ é a quantidade de mão de obra ocupada por unidade de área explorada referente à i-ésima observação (região) e ao ano de $1975(\mathrm{t}=1)$.

$\mathrm{X}_{\mathrm{kil}}$ se refere à k-ésima variável independente, à i-ésima observação (região) e o 1, ao ano de 1975. Os efeitos fixos não mudam com o tempo, por isto $a_{i}$ não apresenta um subscrito indicando o ano.

Considerando uma variável binária $d$ para o ano, sendo $d=0$ (para 1975) e $d=1$ (referente a 2006) e ainda um termo de interação entre $d$ e as variáveis $\mathrm{X}_{\text {kit }}$ tem-se a função da ocupação da mão de obra, em relação ao ano de 2006, como sendo: 
$\ln \mathrm{Y}_{\mathrm{i} 2}=\mathrm{b}_{0}+\delta_{0} d+\left(\mathrm{b}_{1}+\delta_{1} d\right) \mathrm{X}_{1 \mathrm{i} 2}+\left(\mathrm{b}_{2}+\delta_{2} d\right) \mathrm{X}_{2 \mathrm{i} 2}+\left(\mathrm{b}_{3}+\delta_{3} d\right) \mathrm{X}_{3 \mathrm{i} 2}+\ldots+\left(\mathrm{b}_{\mathrm{k}}+\delta_{\mathrm{k}} d\right)$ $\mathrm{X}_{\mathrm{ki} 2}+a_{i}+\mu_{\mathrm{i} 2}$

Neste caso, as variáveis $\mathrm{Y}_{\mathrm{i} 2}$ e $\mathrm{X}_{\mathrm{ki} 2}$ são referentes ao ano de $2006(\mathrm{t}=2)$.

Segundo Wooldridge (2003, cap. 13), para excluir os efeitos fixos, podese subtrair a expressão (1) da expressão (2). Considerando $\Delta \ln Y_{\mathrm{i}}=\ln \mathrm{Y}_{\mathrm{i} 2}$ $-\ln \mathrm{Y}_{\mathrm{i} 1}, \Delta \mathrm{X}_{1 \mathrm{i}}=\mathrm{X}_{1 \mathrm{i} 2}-\mathrm{X}_{1 \mathrm{i} 1}, \quad \Delta \mathrm{X}_{2 \mathrm{i}}=\mathrm{X}_{2 \mathrm{i} 2}-\mathrm{X}_{2 \mathrm{i} 1}, \ldots \Delta \mathrm{X}_{\mathrm{ki}}=\mathrm{X}_{\mathrm{ki} 2}-\mathrm{X}_{\mathrm{ki} 1} \mathrm{e}$ $\Delta \mu_{i}=\mu_{i 2}-\mu_{i 1}$, obtém-se:

$\Delta \ln \mathrm{Y}_{\mathrm{i}}=\delta_{0}+\mathrm{b}_{1} \Delta \mathrm{X}_{1 \mathrm{i}}+\mathrm{b}_{2} \Delta \mathrm{X}_{2 \mathrm{i}}+\ldots .+\mathrm{b}_{\mathrm{k}} \Delta \mathrm{X}_{\mathrm{ki}}+\delta_{1} d \mathrm{X}_{1 \mathrm{i} 2}+\delta_{2} d \mathrm{X}_{2 \mathrm{i} 2}+\ldots+\delta_{\mathrm{k}} d \mathrm{X}_{\mathrm{ki} 2}$ $+\Delta \mu_{\mathrm{i}}$

Assim, os efeitos fixos $a_{i}$ são anulados. O efeito da variável $\mathrm{X}_{\mathrm{k}}$ no ano de 1975 é $b_{k}$ e no ano 2006 é $b_{k}+\delta_{k}$ Além disto, as duas funções diferem pelo valor da mudança no intercepto que é $\delta_{0}$, não se estimando o valor de $\mathrm{b}_{0}$, mas a diferença entre os dois interceptos.

\section{Quadro 1 - Variáveis consideradas na regressão}

\begin{tabular}{|l|l|}
\hline \multicolumn{1}{|c|}{ Código } & \multicolumn{1}{c|}{ Descrição } \\
\hline Lnehpha & $\begin{array}{l}\text { Logaritmo natural do n úmero de equivalentes homem ocupados por } \\
1000 \text { hectares de terra explorada. }\end{array}$ \\
\hline Lavper e lavtemp & $\begin{array}{l}\text { Respectivamente, participação percentual da área de lavouras } \\
\text { permanentes e de lavouras temporárias na área de terra explorada. }\end{array}$ \\
\hline Uva & $\begin{array}{l}\text { Participação percentual da área de uva na área total de lavouras } \\
\text { permanentes. }\end{array}$ \\
\hline $\begin{array}{l}\text { Arroz, feijão, fumo } \\
\text { milho, soja e trigo. }\end{array}$ & $\begin{array}{l}\text { Participação percentual da área de cada uma destas culturas na área } \\
\text { total de lavouras temporárias. }\end{array}$ \\
\hline Gini & Índice de Gini da distribuição de área entre os estabelecimentos \\
\hline Estvin & Percentual de estabelecimentos do município com até 20 hectares \\
\hline Estduz & $\begin{array}{l}\text { Percentual de estabelecimentos do município com mais de 200 } \\
\text { hectares }\end{array}$ \\
\hline Trat & Numero de tratores por 1000 hectares de lavoura \\
\hline Bovpro & $\begin{array}{l}\text { Participação percentual da produção bovina e ovina no valor total da } \\
\text { produção animal }\end{array}$ \\
\hline Suavpro & $\begin{array}{l}\text { Participação percentual da produção suína e de aves no valor total } \\
\text { da produção animal }\end{array}$ \\
\hline
\end{tabular}


Cada uma destas variáveis acima se refere ao ano de 1975 e ao ano de 2006. Assim, nos resultados da regressão apresentados abaixo, cada código do quadro acima é seguido pela terminação 75 e 06. Exemplo, no caso do número de tratores, tem-se: Trat75 e Trat06. Além disto, a variável que representa as diferenças entre os valores de cada variável, de 1975 para 2006, é antecedida pela letra D.

O índice de Gini da distribuição da terra entre os estabelecimentos rurais da região é calculado de acordo com Hoffmann (1998, cap.16). A variável referente à quantidade de tratores por 1000 hectares de lavoura é expressa em termos de tratores equivalentes a menos de $100 \mathrm{CV}$. O censo de 2006 informa o número de tratores de menos de $100 \mathrm{CV}$ e o de mais de 100 $\mathrm{CV}$. Para somar estas duas parcelas, considerou-se o trator de mais de $100 \mathrm{CV}$ equivalente a dois tratores de menos de $100 \mathrm{CV}$.

O valor da produção bovina e ovina (bovpro) é a soma dos valores dos bovinos e ovinos vendidos com os abatidos e a produção de lã, sendo subtraídos os valores dos bovinos e ovinos comprados. Não é incluído o leite por considerar sua produção de características distintas da exploração do gado de corte.

A diferença entre o logaritmo natural do número de equivalentes homem ocupados por 1000 hectares de terra explorada em 2006 e o logaritmo natural da mesma variável referente a 1975 é a variável dependente na regressão.

\section{Resultados}

\subsection{Evolução da ocupação da mão de obra}

O valor de $D$ obtido através do teste de normalidade de KolmogorovSmirnov é igual a 0,066, tanto em relação à distribuição de 1975 como à de 2006. Segundo a Tabela E (SIEGEL, 1956, p.251), as duas distribuições são normais ao nível de $1 \%$ de significância estatística. 
Na estimativa da dimensão do intervalo das classes (valor de $h$ ), obteve-se um valor igual 0,332 referente à distribuição do ano de 1975 e igual a 0,344 para a do ano de 2006. Optou-se por utilizar o valor médio aproximado de 0,34 para cada um dos dois anos. Assim, as duas distribuições de valores relativos (X) dos equivalentes homem por hectare explorado foram divididas em seis classes, que são as seguintes: a) $0<$ $\mathrm{X}<0,32$; b) $0,32 \leq \mathrm{X}<0,66$;

c) $0,66 \leq X<1,00$; d) $1,00 \leq X<1,34$ e) $1,34 \leq X<1,68$; f) $1,68 \leq X$.

Sendo $F_{t}$ e $F_{t+1}$ as distribuições regionais da mão de obra ocupada por hectare explorado, respectivamente no tempo $t$ (1975) e $t+1$ (2006), tem-se o sistema de equações de diferenças que expressa a evolução da distribuição ao longo do tempo. Esse sistema é representado por $F_{t+1}=M$ $F_{t}$ em que $M$ é a matriz de transição de Markov, indicando a probabilidade de cada região da classe $i$ de equivalentes homem por hectare explorado no tempo $t$ estar na classe $j$ no tempo $t+1$. A hipótese básica associada a este procedimento é a de que as probabilidades de transição sejam estacionárias, isto é, que a probabilidade de passagem de uma classe para outra seja invariável no tempo.

A matriz de Markov e o sistema de equações de diferenças resultantes dos dados do estudo são:

$$
\left[\begin{array}{l}
F 1_{t+1} \\
F 2_{t+1} \\
F 3_{t+1} \\
F 4_{t+1} \\
F 5_{t+1} \\
F 6_{t+1}
\end{array}\right]=\left[\begin{array}{cccccc}
0,912 & 0,080 & 0,050 & 0 & 0 & 0 \\
0,088 & 0,717 & 0,050 & 0 & 0 & 0 \\
0 & 0,160 & 0,650 & 0,217 & 0,040 & 0 \\
0 & 0,003 & 0,150 & 0,523 & 0,480 & 0,069 \\
0 & 0,040 & 0 & 0,217 & 0,240 & 0,448 \\
0 & 0 & 0,100 & 0,043 & 0,240 & 0,483
\end{array}\right]\left[\begin{array}{c}
F 1_{t} \\
F 2_{t} \\
F 3_{t} \\
F 4_{t} \\
F 5_{t} \\
F 6_{t}
\end{array}\right]
$$


A solução particular deste sistema de equações de diferenças é

$\left[\begin{array}{l}F 1_{t} \\ F 2_{t} \\ F 3_{t} \\ F 4_{t} \\ F 5_{t} \\ F 6_{t}\end{array}\right]=\left[\begin{array}{cccc}0,0001 & 0,2041 & 0,0167 & -0,0109 \\ 0,0001 & 0,1021 & 0,0063 & 0,0204 \\ -0,0020 & 0,2041 & -0,0041 & 0,0125 \\ 0,0090 & 0,2245 & -0,0088 & -0,0106 \\ -0,0117 & 0,1428 & -0,0055 & -0,0073 \\ 0,0045 & 0,1225 & -0,0046 & -0,0042\end{array}\right]\left[\begin{array}{c}(-0,10387)^{t} \\ (1)^{t} \\ (0,92762)^{t} \\ (0,70257)^{t}\end{array}\right]+$

$$
\left[\begin{array}{cc}
0,0489 & -0,1326 \\
0,1425 & -0,2789 \\
-0,5493 & 1,5727 \\
0,0166 & -0,8837 \\
0,2093 & -0,4768 \\
0,1319 & 0,1993
\end{array}\right]\left[\begin{array}{l}
(0,3884 \cos 3,61 t+0,0832 \operatorname{sen} 3,61 t)(0,49783)^{t} \\
(0,0832 \cos 3,61 t-0,3884 \operatorname{sen} 3,61 t)(0,49783)^{t}
\end{array}\right]
$$

A Tabela 1 apresenta a solução de equilíbrio de longo prazo (quando o tempo $t \rightarrow \infty$ ) do sistema de equações acima em termos de participação percentual das regiões nas seis classes de níveis de ocupação da mão de obra. Na Tabela 1 constam, também, os percentuais de participação das regiões nas classes em 1975 e 2006, bem como as mudanças de 1975 a 2006 e de 2006 ao longo prazo.

Tabela 1 - Participação percentual das regiões nas várias classes de nível de ocupação da mão de obra em 1975, 2006 e no longo prazo e mudanças nos percentuais de um período a outro.

\begin{tabular}{|c|c|c|c|c|c|}
\hline \multirow{2}{*}{ Classes } & \multicolumn{3}{|c|}{ Percentuais } & \multicolumn{2}{c|}{ Mudanças nos percentuais } \\
\cline { 2 - 6 } & 1975 & 2006 & Longo prazo & De 1975 a 2006 & De 2006 ao longo prazo. \\
\hline 1 & 21,79 & 21,79 & 20,41 & 0,00 & $-1,38$ \\
\hline 2 & 16,07 & 14,10 & 10,21 & $-1,97$ & $-3,89$ \\
\hline 3 & 12,82 & 14,74 & 20,41 & 1,92 & 5,67 \\
\hline 4 & 14,74 & 18,63 & 22,45 & 3,89 & 3,82 \\
\hline 5 & 16,03 & 16,03 & 14,28 & 0,00 & $-1,75$ \\
\hline 6 & 18,59 & 14,74 & 12,25 & $-3,85$ & $-2,49$ \\
\hline
\end{tabular}

Fonte: Cálculos dos autores com base em dados dos censos agropecuários do IBGE referentes aos municípios do Rio Grande do Sul e aos anos de 1975 e 2006. 
Portanto, haveria, no longo prazo, uma pequena redução na participação das regiões situadas nas duas classes mais baixas e nas duas classes mais altas de mão de obra ocupada por hectare explorado, enquanto a participação das regiões situadas na classe logo abaixo da média e naquela situada imediatamente acima da média aumentaria no longo prazo. $\mathrm{O}$ maior aumento na participação percentual, de 5,67 pontos percentuais, é na classe de equivalentes homens por hectare, em termos relativos, no nível de $(0,66 \leq \mathrm{X}<1,00)$.

Esta evolução futura na participação das regiões nas classes de ocupação da mão de obra pode ser comparada com o que ocorreu de 1975 a 2006. Neste período, as classes que aumentaram a sua participação são as de níveis $(0,66 \leq X<1,00)$ e a $(1,00 \leq X<1,34)$, portanto, as mesmas classes que aumentarão a sua participação no futuro. Verifica-se também que, se no passado houve redução maior na participação das regiões com elevada ocupação da mão de obra, no futuro, as regiões com pouca ocupação da mão de obra terão uma redução mais intensa.

\subsection{Variáveis explicativas da evolução da ocupação da mão de obra}

Na Tabela 2, são apresentados os resultados do modelo, em que tanto as variáveis em nível quanto as diferenças são utilizadas como explicativas. A variável explicada é a diferença no logaritmo natural do número de equivalentes homem ocupados por 1000 hectares de terra explorada entre 1975 e 2006. 
Tabela 2 - Resultados da regressão estatística da mudança no logaritmo natural do número de equivalentes homem ocupados por 1000 hectares de terra explorada, entre 1975 e 2006, sobre as variáveis abaixo referidas.

\begin{tabular}{|l|c|c|}
\hline Variáveis explicativas & Coeficiente & Probabilidade \\
\hline Constante & 0,1796 & 0,437 \\
\hline Dlavper & $\mathbf{0 , 0 1 9 6}$ & 0,020 \\
\hline Lavper06 & $\mathbf{- 0 , 0 1 6 6}$ & 0,007 \\
\hline Dlavtemp & $\mathbf{0 , 0 0 7 5}$ & 0,001 \\
\hline Lavtemp06 & $-0,0024$ & 0,219 \\
\hline Duva & $-0,0013$ & 0,405 \\
\hline Uva06 & $\mathbf{0 , 0 0 3 0}$ & 0,028 \\
\hline Darroz & 0,0013 & 0,348 \\
\hline Arroz06 & $\mathbf{- 0 , 0 0 4 8}$ & 0,001 \\
\hline Dfeijao & 0,0023 & 0,455 \\
\hline Feijao06 & $\mathbf{- 0 , 0 0 5 2}$ & 0,081 \\
\hline Dfumo & $\mathbf{0 , 0 1 1 8}$ & 0,033 \\
\hline Fumo06 & $\mathbf{- 0 , 0 0 7 9}$ & 0,056 \\
\hline Dmilho & 0,0020 & 0,136 \\
\hline Milho06 & $-0,0018$ & 0,279 \\
\hline Dsoja & 0,0018 & 0,164 \\
\hline Soja06 & $\mathbf{- 0 , 0 0 3 3}$ & 0,032 \\
\hline Dtrigo & $-0,0001$ & 0,954 \\
\hline Trigo06 & $-0,0018$ & 0,649 \\
\hline Dgini & $-0,1586$ & 0,619 \\
\hline Gini06 & $\mathbf{- 0 , 4 6 3 8}$ & 0,038 \\
\hline Destvin & $\mathbf{0 , 0 1 2 5}$ & 0,000 \\
\hline Estvin06 & $\mathbf{0 , 0 0 6 5}$ & 0,000 \\
\hline Destduz & $\mathbf{- 0 , 0 1 8 8}$ & 0,004 \\
\hline Estduz06 & $-0,0033$ & 0,379 \\
\hline Dtrat & $\mathbf{- 0 , 0 0 6 0}$ & 0,000 \\
\hline Trat06 & $\mathbf{0 , 0 0 5 1}$ & 0,000 \\
\hline Dbovpro & $\mathbf{- 0 , 0 0 6 7}$ & 0,003 \\
\hline Bovpro06 & 0,0029 & 0,149 \\
\hline Dsuavpro & 0,0006 & 0,733 \\
\hline Suavpro06 & $-0,0013$ & 0,470 \\
\hline R ajustado & 0,75 & \\
\hline Estatística F & 16,44 & 0,000 \\
\hline & & \\
\hline & & \\
\hline
\end{tabular}

Fonte: Cálculos dos autores com base em dados dos censos agropecuários do IBGE referentes aos municípios do Rio Grande do Sul e aos anos de 1975 e 2006.

*Observações: Conforme os códigos das variáveis, descritos no Quadro 1, a variável dependente é DLnehpha = Lnehpha06 - Lnehpha75. Os coeficientes estatisticamente significantes a $1 \%, 5 \%$ ou $10 \%$ são apresentados em negrito. 
Com base nos resultados apresentados na Tabela 2, a função estimada em relação ao ano de 1975 é a seguinte:

Lnehpha75 $=0,0196$ Lavper75 $+0,0075$ Lavtemp $75+0,0118$ Fumo $75+$ 0,0125 Estvin75-0,0188Estduz75 -0,0060Trat75-0,0067Bovpro75

E a função estimada em relação ao ano de 2006 é a seguinte:

Lnehpha06 $=0,0030$ Lavper06 + 0,0075Lavtemp06 + 0,0030Uva06 - 0,0048Arroz06 -0,0052Feijão06 +0,0039Fumo06 -0,0033Soja06 $-0,4638$ Gini06 + 0,0190Estvin06 -0,0188Estduz06 -0,0009Trat06 $-0,0067$ Bovpro06

Esses resultados mostram que o aumento da participação percentual das lavouras (permanentes e temporárias) na área total de terra explorada tem um impacto positivo sobre a ocupação da mão de obra. Em 1975, um aumento de um ponto percentual na participação das lavouras permanentes na área explorada elevava a ocupação da mão de obra em 1,96\%. Em 2006, este efeito se torna menor, de apenas 0,30\%. Já o impacto da área da lavoura temporária sobre a ocupação da mão de obra se mantém constante, em termos percentuais, de 1975 a 2006, correspondendo a $0,75 \%$ de aumento na ocupação da mão de obra para a elevação de um ponto percentual na participação da área deste tipo de lavoura.

Quanto aos efeitos individuais de culturas específicas, observa-se que em 1975 apenas o fumo apresentava impacto sobre a ocupação da mão de obra. O aumento de um ponto percentual na participação desta cultura na área das lavouras temporárias elevaria a ocupação da mão de obra em $1,18 \%$ em 1975 e de somente $0,39 \%$ em 2006. Isto indicaria uma redução da importância desta cultura para a geração de emprego de mão de obra na agricultura.

Entre as demais culturas testadas, apenas a uva impactou positivamente a ocupação da mão de obra. Em 2006, o aumento de um ponto percentual na participação desta cultura na área das lavouras permanentes aumentaria 
a ocupação da mão de obra em 0,30\%. Além da uva, em 2006, as culturas de arroz, feijão e soja apresentam impactos negativos sobre a ocupação da mão de obra, respectivamente, de $-0,48 \%,-0,52 \%$ e de $-0,33 \%$ para cada aumento de um ponto percentual na participação da cultura correspondente na área das lavouras temporárias. Isto pode ser explicado pelo fato de estas culturas, especialmente o arroz e a soja, serem altamente mecanizadas.

A mecanização das lavouras, de acordo com a expectativa, reduz o emprego da mão de obra. Esse resultado é consistente com os resultados encontrados por Irwin et al. (2010) para os Estados Unidos e também por Stabler e Olfert (2009) para o Canadá. O efeito dos tratores reforça esse resultado, mostrando que o aumento de um trator por mil hectares de lavoura reduz o emprego da mão de obra em $0,60 \%$ e $0,09 \%$, respectivamente, em 1975 e 2006. Percebe-se que o impacto negativo dos tratores diminuiu durante o período analisado. Isto talvez se deva ao fato de o número de tratores haver aumentado muito no Rio Grande do Sul entre 1975 e 2006, de 14 para 30 tratores/mil hectares de lavoura, em termos de tratores equivalentes a menos de $100 \mathrm{CV}$.

Outra atividade com efeito significativo sobre o emprego da mão de obra é a produção bovina e ovina. $\mathrm{O}$ aumento em um ponto percentual do valor da produção bovina (exclui o leite) e ovina no valor total da produção animal reduziria a ocupação da mão de obra em $0,67 \%$, tanto em 1975 como em 2006. Este resultado pode ser devido às características da criação de forma extensiva, sem necessidade de emprego de muita mão de obra.

A participação dos estabelecimentos rurais com até 20 hectares de área impacta positivamente a ocupação da mão de obra. O aumento em um ponto percentual na participação destes estabelecimentos em relação ao total elevaria a ocupação da mão de obra em 1,25\% e 3,15\%, respectivamente, em 1975 e 2006. Portanto, aumentou muito, neste período, a importância dos pequenos estabelecimentos rurais para a ocupação da mão de obra. 
A participação dos estabelecimentos rurais com mais de 200 hectares tem um efeito negativo sobre a ocupação da mão de obra. O aumento em um ponto percentual na participação destes estabelecimentos reduziria a ocupação da mão de obra em 1,88\%, tanto em 1975 como em 2006.

A participação do número de estabelecimentos de menos de 20 hectares no total dos estabelecimentos rurais aumentou de $60,64 \%$ para $65,38 \%$ de 1975 a 2006. Porém, a participação destes estabelecimentos no total da área somente variou de $10,92 \%$ para $11,53 \%$, indicando que o número deles aumentou, principalmente, pela divisão da área dentro do próprio segmento. Isto fica mais evidente quando se observa que o percentual do número de estabelecimentos com menos de 10 hectares variou mais, de $32,60 \%$ para $39,48 \%$.

Entre 1975 e 2006, a participação do número de estabelecimentos rurais com mais de 200 hectares variou apenas de $4,04 \%$ para $4,10 \%$, e sua participação na área total aumentou apenas de 56,72\% para 57,88\%.

Outra variável com impacto sobre o nível de ocupação da mão de obra é o índice de Gini da distribuição de área dos estabelecimentos. Porém, este efeito somente se observa em relação ao ano de 2006. Uma redução de 0,01 no índice de Gini aumentaria a ocupação da mão de obra em $0,46 \%$.

\section{Conclusões}

O Rio Grande do Sul é um estado com uma produção agropecuária bastante diversificada. As regiões de pecuária extensiva utilizam pouca mão de obra por área explorada, assim como as regiões produtoras de soja, trigo e arroz onde há uma grande mecanização. Porém, nas regiões minifundiárias, com uma produção diversificada, a ocupação da mão obra por área explorada é elevada. Nestas regiões, a pequena dimensão do estabelecimento rural torna inviável economicamente a utilização de tratores. 
A análise de convergência entre regiões, classificadas em seis níveis de ocupação da mão de obra por área de terra explorada, mostrou que, no futuro projetado com base no histórico de 1975 a 2006, a classe das regiões com um nível de ocupação da mão de obra entre a média do Estado e $66 \%$ desta média aumentará sua participação em 5,67 pontos percentuais e a classe logo acima da média do Estado aumentará sua participação em 3,82 pontos percentuais. As demais classes terão sua participação diminuída.

Dada a redução na participação por parte das duas classes mais baixas de ocupação da mão de obra, pode-se especular que algumas das regiões, destas classes, com criação de gado de forma extensiva passem aos poucos para um processo um pouco mais intensivo em mão de obra. Da mesma forma, algumas regiões com altos níveis de mecanização podem diversificar um pouco a produção, na direção de produtos que requerem mais mão de obra.

Por outro lado, a pequena redução na participação das regiões situadas nas classes mais elevadas de ocupação da mão de obra indicaria um processo de substituição parcial da mão de obra por capital em apenas algumas delas.

Com base nos resultados das regressões, é possível concluir que a utilização de mão de obra nas regiões do Rio Grande do Sul apresenta comportamento heterogêneo, de acordo com o tipo de cultivo e estrutura fundiária predominante. Os fatores que influenciam as variações na quantidade de mão de obra estão de acordo com o esperado e são consistentes com o comportamento verificado em países como Estados Unidos e Canadá. No que tange à produção agrícola, fica evidente que a presença de culturas mais propensas à mecanização impacta negativamente a utilização de mão de obra, e as culturas familiares, de produção intensiva como fumo e uva, contribuem para aumentar a utilização de mão de obra. Por sua vez, a pecuária extensiva, como bovinos e ovinos, também contribui para reduzir a quantidade demandada de mão de obra. 
Em síntese, os estabelecimentos pequenos, destinados a lavouras diversificadas, seriam os maiores responsáveis pela maior retenção do trabalhador no meio rural. Os estabelecimentos rurais maiores, que possibilitariam uma maior substituição do homem pelo capital (mecanização), ou então, destinados a uma criação mais extensiva de gado, não reteriam o trabalhador rural no mesmo nível.

Ressalta-se que as projeções sobre a convergência das regiões entre as classes de níveis de ocupação da mão de obra foram realizadas com base no histórico ocorrido entre 1975 e 2006 . Pode ocorrer que, no futuro, devido a maiores oportunidades de emprego nas cidades, com salários mais atrativos, resultantes de um maior desenvolvimento dos setores industrial e de serviços, haja uma maior migração do trabalhador rural para a cidade. Com a menor disponibilidade da mão de obra no meio rural, poderia ocorrer a agregação dos pequenos estabelecimentos, possibilitando-lhes uma maior mecanização, o que reduziria os níveis de ocupação da mão de obra também nos pequenos estabelecimentos.

Contudo, enquanto não houver mais emprego e existirem problemas de infraestrutura no meio urbano, a retenção do trabalhador no meio rural, principalmente pelos pequenos estabelecimentos, é importante para um maior bem-estar da população.

\section{Referências}

GUERREIRO, E. Produtividade do trabalho e da terra na agropecuária paranaense. Revista de Economia e Sociologia Rural. v. 34, n.1, SOBER. 1996. (Artigo acessado no CD disponibilizado pela SOBER).

HARRIS, J.; MICHAEL, T. Migration, unemployment, and development: A two-sector analysis. American Economic Review, v.60, p.126-142, 1970.

HOFFMANN, R. Estatística para Economistas. São Paulo: Biblioteca Pioneira de Ciências Sociais. 1998. 
IRWIN, E. G.; ISSERMAN, A. M.; KILKENNY, M., PARTRIDGE, M. D. A century of research on rural development and regional issues. American Journal of Agricultural Economics. v.92, n.2, p.522-55. April 2010.

LEWIS, W. A. Economic development with unlimited supplies of labour. Manchester School of Economic and Social Studies, 22, p. 131-191, 1954.

LUCAS JR., ROBERT, E. Life earnings and rural-urban migration. Journal of Political Economy. v. 112, n. S1. p. S29-S59. February, 2004.

MAGRINI, S. The evolution of income disparities among the regions of the European Union. Regional science and urban economics, $n$. 29, 1999.

PAGAN, A.; ULLAH, A. Nonparametric Econometrics. Cambridge/ UK: Cambridge University Press, 1999.

SIEGEL, S. Nonparametric Statistics for the Behavioral Sciences. Nova Iorque: McGraw-Hill Book Company, 1956.

TODARO, M. P. A Model of Labor migration and urban unemployment in less developed countries, American Economic Review, 60, p. 138$148,1969$.

VAIDYANATHAN, A. Labour use in rural india: A study of spatial and temporal variations. Economic and Political Weekly, v.21, n. 52 (Dec. 27, 1986). Pp. A130-A133+A135-A137+A139-A146.

WOOLDRIDGE, Jeffrey M. Introductory econometrics. ThomsonSouth-Western - Estados Unidos- 2003

WORLD BANK. World Development Report 2010: Development and Climate Change. 2010. 